\title{
Civilisations
}

Revue internationale d'anthropologie et de sciences

humaines

44 | 1997

Les peuples des forêts tropicales

\section{Économie et impact de l'agriculture itinérante Badjoué [sud-Cameroun]}

\section{Pauwel de Wachter}

\section{OpenEdition \\ Journals}

Édition électronique

URL : http://journals.openedition.org/civilisations/1611

DOI : 10.4000/civilisations. 1611

ISSN : 2032-0442

\section{Éditeur}

Institut de sociologie de l'Université Libre de Bruxelles

\section{Édition imprimée}

Date de publication : 1 janvier 1997

Pagination : 62-93

ISBN : 2-87263-122-4

ISSN : 0009-8140

Référence électronique

Pauwel de Wachter, «Économie et impact de l'agriculture itinérante Badjoué [sud-Cameroun] », Civilisations [En ligne], 44 | 1997, mis en ligne le 29 juin 2009, consulté le 19 avril 2019. URL : http:// journals.openedition.org/civilisations/1611; DOI : 10.4000/civilisations.1611 
ÉCONOMIE et IMPACT de l'AGRRCULTURE ITINËPRTIE bRDJUUE [SUD-CAMEROUN]

Pavwel de WhCHTER 


\section{INTRODUCTION}

Cet article concerne des essarteurs d'Afrique Centrale qui vivent dans un environnement caractérisé par des ressources abondantes et dans lequel la production vivrière est liée étroitement à l'investissement en travail humain. Ces conditions d'abondance sont encore présentes dans de grandes parties forestières d'Afrique Centrale ${ }^{[l]}$.

Nous étudions de façon quantitative les différents types de champs qui correspondent à la mise en culture de différents types de jachères. Des données quantifiées fiables nous permettent de quantifier l'impact actuel et futur de l'essartage sur la forêt. Il s' avère que l'essartage Badjoué n'est actuellement pas une menace pour les forêts primaires du Dja.

Le paysage créé par l'essartage Badjoué est une mosaïque de forêts primaires et secondaires capable de soutenir une pression de chasse plus élevée que la forêt primaire.

Ce travail s'inscrit dans le cadre d'une recherche réalisée pour le projet ECOFAC-Cameroun. Ce programme, financé par l'Union Européenne, organise la gestion de la Réserve de Faune du Dja selon une politique de gestion participative.

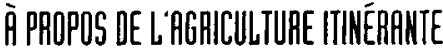

Lagricullure itinérante traditionnelle dans une zone à faible densité de population est aujourd'hui considérée comme un système agraire durable du point de vue écologique [National Research Council, 1993; Sanchez, 1976; Whitmore, 1992; Beets, 1990; Kang e.a. 1984].

Néanmoins dans beaucoup de textes et de statistiques, l'agriculture itinérante est accusée, de façon parfois peu nuancée, comme très destructive pour les forêts tropicales. Je cite: « Aujourd' hui, cependant, l'agriculture sur brûlis est dénoncée comme un facteur décisif de la destruction croissante des sols agricoles, de l'érosion, de la désertification et de l'appauvrissement généralisé des pays et des régions où elle se pratique » [Gutelman, 1989].

Nous pouvons apporter les nuances suivantes:

1. l'essartage correspond aux besoins primaires (produire la subsistance végétale de base et, si possible, gagner un petit revenu monétaire) de la population concernée et ainsi diffêre des autres causes de déforestation. Les autres causes sont les plantations industrielles, l'exploitation forestière et son effet secondaire de création des voies d'accès à la forêt, les activités minières, les projets d'infrastructure (routes, barrages). Ces activités répondent aux besoins des secteurs «modernes» de l'économie;

2. en l'absence de croissance démographique, l'essartage traditionnel est peu consommateur des forêts primaires. Mettre en culture une forêt primaire plutôt qu'une forêt secondaire adulte n'offre guère d'avantages agricoles mais demande plus de travail d'abattage;

3. en ce qui concerne la croissance démographique, il s'agit de savoir si l'agriculture itinérante peut être remplacée par des systèmes agraires moins consommateurs de terre. Pour des raisons d'économie du travail et d'opération dans un environnement riche en ressources, ceci n'est pas une éridence.

Le facteur de production limitant, dans le système de production dont l'essartage fait partie, est 


exploitation des ressources, il s'avère nécessaire de donner un statut de protection à une partie de la forêt et de freiner les causes de l'immigration. Un des défis urgents pour le futur est de trouver des modèles de gestion d'aires protégées qui prennent en compte les aspirations des essarteurs traditionnels résidents. C'est dans cet esprit que le projet ECOFAC organise, selon une politique de gestion participative, la gestion de la Réserve de Faune du Dja.

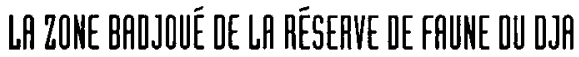

La Réserve de Faune du Dja $\left(5260 \mathrm{~km}^{2}\right)$ est située à $230 \mathrm{~km}$ au sud-est de Yaoundé. Elle est située dans la boucle du Dja. Cette rivière fait partie du bassin du fleuve Zaïre. La région a un climat équatorial toujours chaud avec un régime pluviométrique bimodal. Il pleut environ 1640 $\mathrm{mm} /$ année, reparti en deux saisons de pluie (15 mars - 15 juin et 15 août - 15 novembre). La végétation dominante de la Réserve est la forêt dense humide sempervirente. Le relief y est peu marqué et le site se trouve entre $600 \mathrm{~m}$ et $700 \mathrm{~m}$ d'altitude. L'aire protégée est entourée par des forêts qui sont vouées en premier lieu à des activités d'exploitation forestière pour le bois.

La zone Nord de la Réserve est habitée par une population résidente permanente d'environ 1300 personnes de l'ethnie Badjoué [ECOFAC, 1993a]. Les zones au Sud de la partie Badjoué sont inhabitées (quoique visitées par des Baka peu nombreux). Cette population Badjoué de la Réserve vit dans 17 villages le long d'une piste ouest-est qui mesure $46 \mathrm{~km}$ à vol d' oiseau (trajet Somalomo-Ndengue carrossable jusqu'à Ekom). La densité linéaire de population le long de cette piste équivaut à $28 \mathrm{hts} / \mathrm{km}$. La grande partie des activités agricoles (essartage, plantations de cacao et de café) est située au maximum à $5 \mathrm{~km}$ de la piste et des villages ${ }^{[b]}$. Ainsi, la superficie des terres suffisamment proches pour l'agriculture correspond à $460 \mathrm{~km}^{2}$. Il en suit une densité de population de $2.8 \mathrm{hts} / \mathrm{km}^{2}$. Nous avons estimé la superficie des forêts sur sols hydromorphes à $33 \%{ }^{[7]}$ de la zone suffisamment proche pour l'agriculture (sur base d'un échantillon de $95 \mathrm{~km}^{2}$ le long de $13 \mathrm{~km}$ de cette piste couvert par des photos aériennes $\left.(1 / 20.000)^{[8]}\right)$. Ensuite, nous obtenons une superficie de $308 \mathrm{~km}^{2}$ de terres suffisamment "proches" et ayant des sols avec bon drainage ${ }^{[(])}$. La densité de population par rapport à cette dernière superficie correspond à $4.2 \mathrm{hts} / \mathrm{km}^{2}$. Cette dernière densité est actuellement déterminante en ce qui concerne les terres utilisables pour l'agriculture et pour l'emplacement actuel des villages. La population de la réserve est engagée dans un système de production varié qui comporte l'essartage, les cultures pérennes de cacao et de café, la chasse et la pêche (de subsistance et commerciale) et la cueillette. Les céphalophes sont le principal gibier chassé, et parmi la grande variété des produits soumis à la cueillette, citons les graines du moabi (Baillonella toxisperma) pour la fabrication d'huile et les amandes d'Irringia gabonensis pour enrichir les săuces.

Le terroir forestier des villages s'étend bien au-delà du rayon de $5 \mathrm{~km}$. Dans le village échantillon d'Ekom les lignes de pièges s'étendent jusqu'à $20 \mathrm{~km}$ du village [Dethier, 1995, Joiris et Tchikangwa, 1995]. Des chasseurs d'autres villages à l'Est de la Réserve pénètrent même jusqu'au moins $40 \mathrm{~km}$ en forêt pour établir des 

lignes de piège. Ces lignes de piège fournissent le commerce de viande boucanée.

Par ailleurs, des plantations continuent parfois d'être exploitées à l'emplacement d'un ancien village.

Le projet ECOFAC qui organise la gestion de la RFD a choisi Ekom pour une étude de cas incluant des recherches anthropologiques [Joiris et Tchikangwa, 1995], de chasse [Dethier, 1995] et agricoles [de Wachter, 1995]. Le village d'Ekom se trouve à l'extrême est de la partie carrossable de la piste et a une population résidente de 154 habitants, ce qui est environ un huitième de la population de la zone Nord de la Réserve de Faune du Dja. Ekom et les villages avoisinants Koungoulou, Bodjouo et Noo regroupent environ un quart de la population de la zone Badjoué de la Réserve. Ainsi, c'est un des endroits les plus peuplés de la Réserve.

Nous décrirons de façon synthétique les types de champs et la taxonomie des jachères Badjoué. Puis, nous essayerons d'expliquer les modalités d'utilisation des jachères. La répartition du défrichement annuel en types de jachères nous permet d'estimer le cycle moyen culture-jachère de l'agriculture Badjoué. Nous obtenons ainsi le besoin minimal en terres pour l'agriculture itinérante actuelle. Nous pouvons également anticiper sur les besoins futurs en terre.

\section{LES TYPES DE CHAMPS VIVHIEAS}

(cf. en annexe le tableau résumant les données quantitatives)

Nous pouvons distinguer deux classes de champs chez les Badjoué: les champs diaracbides et associés qu'on établit sur jachère de courte durée ( 4 ans de durée de jachère au moment de la mise en culture) et moyenne durée (11 ans en moyenne) et les champs sans aracbides, où le bananier plantain et/ou le concombre dominent. Ces derniers champs sont établis sur une forêt secondaire adulte (20 ans et plus de durée de jachère). Les champs de type arachide occupent $75 \%$ des surfaces défrichées dans l'année et les champs sans arachides occupent les autres $25 \%$ de la superficie totale mise en culture par an. Les champs de type arachide sont donc les plus importants en termes de superficie.

Champs de type «arachide»: la matrice culturelle (cultures associées) qui domine le champ est constituée d'arachides, de manioc et de maiss, et, sur jachère forestière, de plantain et de concombre (Cucumeropsis mannii; seulement premier cycle). C'est un champ obligatoire pour le ménage Badjoué parce que les arachides sont un ingrédient journalier dans la cuisine (la première source de lipides et protéines végétaux). La parcelle moyenne a une superficie moyenne de 0.21 ha. Le champ diaracbides du premier cycle (38\% des surfaces annuelles, 1 parcelle par femme cultivatrice par anl) est ensemencé et planté en mars-avril (après la grande saison sèche). Le champ d'arachides du deuxième cycle ( $37 \%$ des surfaces annuelles, 0.92 parcelles par femme cultivatrice par an) est mis en culture en août-septembre (après la petite saison sèche).

La superficie couverte par les arachides est en moyenne de $79 \%(n=11, s=5 \%)$. Quand on additionne les touffes de manioc et les pieds de plantain, on obtient une densité moyenne de 1852 unités/ha pour le champ d'arachides sur jachère préforestière et 2698 unités/ha pour les 

















swinderianus)) peut renforcer cette tendance. Ceci sera une façon économiquement saine de valoriser des surplus de manioc.

Dans le futur, avec la croissance de la population, le complexe co-adaptatif zones agricolesfaune déprédatrice deviendrait plus important dans l'écosystème et en même temps la nécessité d'une bonne gestion des ressources cynégétiques deviendra plus urgente.

\section{CONCLLSION}

L'essartage influence seulement la zone le long de la piste Somalomo-Ndengue. Les vieilles forêts de l'intérieur de la Réserve ne sont pas affectées par les activités agricoles contemporaines. Il se pourrait que l'effet écologique soit même positif parce que l'écosystème résultant de l'essartage est plus varié que la forêt homogène. Dans la forêt primaire, les ouvertures qui se créent sans l'intervention de l'homme sont petites (les chablis sont rarement plus grands que $500 \mathrm{~m}^{2}$ ). C'est seulement dans le centre de tels grands chablis que le parasolier peut s'établir [Hartshorn in Hamilton, 1989]. Les ouvertures créées par l'agriculture Badjoué à distance des villages varient entre 10 ha et 50 ha. Avec des longs cycles agricoles et avec le phénomène d' abandon de certaines zones de jachères. ces ouvertures créent des biotopes qui ne pourraient pas exister dans la forêt primaire sans activité humaine. Cette mosaïque peut probablement soutenir une pression de chasse plus forte que la forêt primaire homogène.

Labsence de certains (gros) mammifères aux environs des villages a été notée par Wilkie \& Finn [1990] (okapi, céphalophe à dos jaune, panthère), Thomas [1991] au Zaïre [Ituri] (chimpanzé et Cercopbitecus bamlyni) et Lahm [1993] au Gabon (chimpanzé, gorille, éléphant, buffle de forêt). Dans la boucle du Dja, nous avons observé l'extinction écologique aux environs du village ${ }^{[33]}$ du gorille, de la panthère, de l'éléphant, du pangolin géant mais le sitatunga et le céphalophe à dos jaune [Dethier, 1995] sont encore présents.

Il faut évaluer l'essartage du point de vue de l'agriculteur. Celui-ci n'est pas un demandeur d'innovations techniques qui augmentent la production par ha tant que la terre est abondante. Ceci se comprend dans le cadre d'une économie basée sur le travail humain et opérant dans un environnement riche en ressources avec un large spectre de possibilités d'investissement du travail. Les projets de développement et les services agricoles ont tendance à offrir aux agriculteurs des innovations agricoles qui économisent la terre mais augmentent le travail (et la complexité de gestion) par unité produite. Or, il n'y a pas de demande pour ce type d' intervention et ces initiatives sont souvent vouées à l'échec. Il vaudrait mieux canaliser les ressources d'un projet dans la création de motivations pour la conservation de la biodiversité (développer l'écotourisme, la gestion de la chasse) et anticiper l'impact futur de la croissance de la population (développer le piégeage autour des champs, enrichir le terroir villageois avec des arbres utiles). 





\section{REMEECEIEMENTS}

Je remercie le professeur $\mathrm{E}$. Tollens. J.P. Vautherin, M. Dethier et spécialement D.V. Joiris pour leurs commentaines. Enfin, je remercie le MINEF, ECOFAC ainsi que J.M. Froment, P. Seme, le professeur W. Delvingt et le professeur J. lejoly pour leur soutien et confiance.

\section{NOTES} 1

Soit le Cameroun, le Gabon, le Congo, la Centrafrique et la Guinée Equatoriale. Les mêmes conditions existent également dans une grande partie du Zaire forestier.

\section{- 2}

Des études de l'IITA sur l'agriculture en zone humide au Cameroun (région de Mbalmayo) ont montré que la culture en couloirs n'est pas attractive pour le paysan quand il a des parcelles fertiles de forêt secondaire à cultiver (IITA, 1993), on peut en conclure que, là où la terre est encore abondante, la culture en couloirs n'économise probablement pas le facteur de production travail par unité de production. Dans des régions où les terres deviennent rares (ex. autour des villes et dans les régions à densité relativement élevée de population), la culture en couloirs est intéressante parce qu elle économise la terre. De plus, c'est une technologie qui demande peu d'intrants ( alow external input agriculture") et qui crée des retombées concrètes pour les agriculteurs dont les faibles movens ne permettent pas d'assumer des risques financiers.
3

Huybens et Tollens (1987) ont pendant une année suivi des exsarteurs Turumbu dans la région de Yangambi au zäre. Ils ont noté l'utilisation du temps entre 6 heures le matin et 6 heures le soir chez les hommes, lagriculture occupait seulement $11 \%$ de leur temps par rappont à $19 \%$ pour des activités extractives. Chez les femmes, l'agriculure occupait $27 \%$ de leur temps et les activités extractives seulement $4 \%$. Les autres catégories d'utilisation du temps étaient les loisiss (h: $45 \%, \mathrm{f}: 29 \%$ ), les activités ménagères (h: $2 \%, \mathrm{f}: 23 \%$ ), lartisanat et la fabrications d'outils (ex. pirogues, maisons) (h: $12 \%, \mathrm{f:} 2 \%$ ), les activités sociales (h: $6 \%, \mathrm{f}: 7 \%$ ), et les repos de maladies (h: $5 \%$, f: $8 \%$ ).

4

lassociation des ferralsols, acrisols, alisols et plinthosols (taxonomie FA) des sols) représente environs $45 \%$ des sols tropicaux (1)riessen \& Dudal. 1991). La fertilité de ces sols acides n'est pas élevée. Ies ferralsols sont dominants sur les vieilles surfaces en Afrique Centrale et Antérique Látine. Les ferralsols ont des bonnes qualités physiques (peu érodibles) mais leurs qualités chimiqutes sont paurrex. Le maintien de la matière organique avec des longues périodes de jachères, le compostage ou le mulch est une technique de gestion agricole importante.

\section{T. 5}

D'autras aspects (projets routiers, colonisation subventionnéc, développement des régions «sous-peuplées" (?!) peuvent causer ou faciliter cette migration. li la démographie galopante de l'Afrique. l'émigration vers les régions peu peuplées sera une importante cause de déforestation dans le siècle à venir.

\section{6}

Les zones agricoles situées relativement loin (ex. $5 \mathrm{~km}$ ) des villages sont centrées sur la culture cacáoyère, qui exige de bonns sols (souvent des anciens fluvisols à proximité du Dja). Les groupements de plantations cacaoyères sont entourés par des jachères, qui reflètent une agriculture vivrière importante datant de la période des bons prix pour le cacao (avant 1989). On y trouve des calanes permettant une résidence sur place.

i) après ces photos aériennes, $7 \%$ de la superficie totale sera couverte avec des raphiales ( $21 \%$ des formations végétales sur sols hydromorphes).

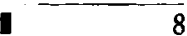

Des photos aériennes de 1989 couvrent la zone le long de la piste Somalomo-Fkom. Line carte forestière a été établie sur base de ces photos (ECOFAC, 1993b).

\section{9}

Les Badjoué n'utilisent pas les forêts sur sols hydromorphes parce que les cultures principales de longue durée (manioc, plantain. macabo) ne supportent pas un sol avec mauvais drai nage. Ils font parfois des petits champs dans les marécages pendant la grande saison sèche. On y met du mais et de la canne à sucre. Mais ce type de champ est très rare. 
10

On y trouve les herbacées Chromolaenu odorala, Afromomum sp., Mikoma augata, Lantema camara, Musa sp., Combretum sp., Costus sp., Orena labata et des semences

d'arbres héliophiles comme Musunga cecropioides, Albizia sp., Ficus micuso, Macaranga spinasa, Rauvolfia macrophilla, Funtumia elastica, Tetrorcbydium dichmostemon, Vernomia coupbenta. Marratiteria discoitdea. Runu olfiu romitoria, Harunga matkiguscariensis, ... Des repousses de souches sont aussi un élément caractéristique de ce recrû forestier.

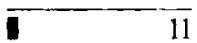

La distinction entre ku alkomo et parasoleraie n'est pas toujours claire pour les cultivateurs parce qu' une bonne partie des kualkomo mises en culture sont des vieilles parasoleraies.

- -12

Il s'agit du moustac (Cercopitbecus cepbus) et du cercocèbe agile (cercocebus galeritus).

$\square-13$

Pour le Sarawak (Malaisie), Cramb (1993) estime que le défrichenent de la forêt primaire représente moins de $5 \%$ de la superficie défrichée par année par les essartetrs. $\sqrt{14}$

Ies féculents: le macabo (Xanthosoma sagittifolium), taro (Colocasia esculenta), la patate douce (/pomoea batatas), l'igname (Dioscorea spp). et la jomme de terre (Solamum tuberosum). Les légumes-feuilles: l'amarante (Amaranthus stp).), la morelle noire (Solanum nigrum). Les légumes-fruits: le melon (Cucurbila s.). l'auhergine amère (Solarium esculentum), le gombo (Hibiscus esculentus), la lomate-cerise (I:Aco persicum esculentum), la mordle amere (Solanum atbiopicum) et le piment (Capsicum frutescons). Enfin il y a le tabac (Nicotiana tabacum) la canne à sucre (Xicharrum sp.), l'haricot (Phaseolus vulgaris), la banane douce (Musa spp) ) et l'oignon (Allium ceph). (es plantes se cultivent daus les chan ops de type "arachides". là ou on a brûlé des tas de debris. Ies tas de debris résultent du nettoyage (enlever les branchettes non-brúlées) du champ d'arachidos. Nous avons estimé le nombre de tas de débris à 165 tas/ha ( $n=18$ champs).

\section{$\overline{1}-\overline{15}$}

lá valeur sur pied est une fonction de l'utilité et de la rareté. Ainsi Baillonella kxisyerma et /ruingia gabononisis seront maintenus parce qu'ils sont rares et très utiles. Ies fruits du Ricinodendrom betidelotli sont appréciés pour les sauces. Cet arbre est utile mais il n'est pas rare et on l'abattra souvent. Son bois très tendre pourrit vite et les agriculteurs estiment que le Ricinodendron abattu rend la terre plus fertile.

- - -16

Dans certains champs sur jaclère préforestière, on arrête la récolte du manioc avant que celui-ci ne soit épuisé. Le Cbromolaena odurata rend la parcelle tellement impénétrable que la cultivatrice préfere récolter du manioc dans un champ plus jeune.

\section{7}

La moyenne de fin des récoltes régulières sur une microsurface aléatoire de la parcelle peut définir catte durée.

\section{8}

La plantation du manioc (dans les champs de type "arachides") a surtout licu entre le 15 mars et le 15 avril (prenier chanu d'arachides) ct entre le 15 août ct le 15 septembre (deuxième chanıp d'arachides).

\section{- 19}

Ie Chromolaena odorata empêche une réinstallation rapide de la forêt sur les parcelles dont la jachère précédente était de conirte durée. Il est donc probable que la reconstitution totale de la fertilité du sol soit ralentie par le Chromolaena puisqu une reconstitution totale de la fertilité demande une végétation variée. Le rétablissement de la richesse minérale est un effet de la diversité. C'ertaines espèces stockent dans leur biomasse plutôt du phosphore, d'autres du potasse, du magnésium, etc. Ie Cbromolaena, avec son enracinement peu profond, ne peut donc pas reconstituer la fertilité totale du sol.

\section{0}

Ceci signifie, dans une situation d'équilibre, que l'âge moyen de la végétation des jachères équivaut $(17 / 2=)$ à 8.5 ans. 
21

93\% des champs vivriers d' Ekom (1993-1994) se situent à moins de deux kn du village. Ies autres clranps vivriers sont situés dans des zones cacaoyères. Avec la reprise de la culture cacaoyère, dès 1994, nous pouvons supposer que la partie des champs situés dans les cacaoyères soit en augmentation. Spécialement le champ d arachides du deuxième cycle (semailles en septembre) est situé près des cacaoyères parce que les semailles, plantation et premier sarclage coüncident avec la récolte du cacao. Les phanteurs ont aussi intérêt à avoir un maximum de présence hunaine dans les zones cacaoyères afin d'effrayer les prédateurs sauvages.

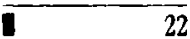

Pour calculer les hesoins en terre il faut multiplier la surface mise en culture par année avec le cycle moven culture-jachère. On fait les calculs par fenme cultivaltice, par homme actif (et défricheur) et pour la zone Nord de la Résenve du Dja (facteur 8 d'extrapolation par rapport à Ekom).

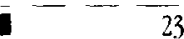

Vu que la composition des ménages est très variable, nous préférons les calculs par femme cultivatrice. Une fernme cultivatrice est une femme qui a résidé à Ekom (le village échantillon) pendant au moins la moitié de la période 1993-1994 et qui a cultivé au moins un champ.

I. 24

À comparer avec Champaud (1973) qui obtient 0.42 ha par femme active chez les Bassa (Sud-Cameroun). Chez les fang (Sud-Cameroun, NordGahon) Alexandre et Binet (1958. dans Joiris et Bahuchet (1993)) obtiennent 0.52 ha par femme active, ce qui est très proche de notre chiffre de 0.55 ha. $\overline{25}$

Il faut tenir compte d'une nouvelle répartition entre tyjes de végétation mis en culture: jachère préforestière $25 \%$, jeune forêt secondaire $21 \%$, forêt secondaire adulte $44 \%$ et forêt "primaire" $10 \%$.

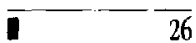

A Ekom chaque planteur possede en movenne 1.4 ha de cacao ì valoriser (1 ha/homme actif). L'évolution des superficies des cultures pérennes dans la boucle dépendra surtout des prix. Dans le futur. au cacav et caté peut s'ajouter le palmier à huile.

\section{7}

Des plantations qui sont en état suffisamment bon pour être travaillées. En 1994, la houcle sortait diune profonde crise du café et du cacao (baisse des prix. problème de transport des récoltes). Une partie seulement de cette superficie est réellement trávaillée.

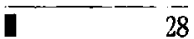

Le cacao était aussi planté conme marqueur de terre (Leplaideur \& al. 1981). Les tiges restent sur place pendant 50 ans et ainsi le cacalo marque la terre pour le planteur et ses héritiers. Ceci explique pourquoi certains planteurs ont plusieurs (parfois 4 ou 5) cacaovères. Cellex-ci ne sont pas travaillées de façon intensive. Une année on travaillait telle cacaoyère et lautre année une autre.

\section{9}

1.a Banqque Mondiale prévoit une croissance de $3.5 \%$ pour la décennie 1990-2000 (World Bank, World Development Report 1992)
30

Un couple est rentré de Yaoundé et 5 élèves de 15 ans ou plus ne sont plus rentrés à l'école en ville parce que les moyens financiers manquaient.

\section{1}

Le contenu en matière sèche $(51 \%$ $26 \%$, Westphal (1985)) des racines fraiches varie selon la période de l'année. Dans le Dja. il y a une longue saison sèche qui dure 4 mois. Pendant cette saison sèche, les tubercules perdent du poids frais, mais le pourcentage en amidon augmente. Le manioc perd aussi de ses feuilles pendant la saison sèche. Quand les pluies reviennent au mois de mars, la plante produit d'abord des nouvelles branches et feuilles et il se peut que la gurantité totale de matière sèche dans les tubercules diminue (Silvestre, 1989). les tuhercules grandissent de nouveau quand tout le feuillage a été établi. La très bonne production de manioc (sélection 1) est du manioc qui a été pesé vers la fin de la période humide (la saison sèche connmence vers minovembre), quand le contenu en eau ext maximal. Il est donc conseillé d'établir la productivité de manioc en utilisant l'unité «matière sèche».

\section{32}

Notons que le piège-barrière attrape surtout des rongeurs et des reptiles alors yue les pièges classiques posés en forêt attrapent surtout des artiodactyles. Avec le piège-barrière on exploite donc une autre ressource. 33

C'est-à-dire que l'espèce n'a plus un rôle important dans l'écossystème. 


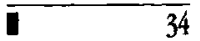

Source: un inventaire de tous les champs vivriers d'Ekom pendant la période 1993-1994 a été établi. Le recensement a été fait avec des interviews de profondeur. Ainsi 189 parcelles et leur jachère d'origine ont été recensées. 151 ( $80 \%$ du total) de ces parcelles ont été visitées et cartographiées.

\section{5}

Peme mode se traduit par champ de nourriture. Des féculents comme le manioc, le plantain et le macabo sont désignés par les Badjoué comme "nourriture». Ici dans le tableau, peme mode signifie un champ avec du plantain, du manioc et du macabo et, éventuellement, du concombre. Ces cultures ont été mises en place sans mettre des arachides (suite par exemple à un manque de semences d'arachides ou parce qu'on n'a pas eu le temps d'eusemencer le champ en arachides).

\section{6}

Une femme cultivatrice est une femme résidente qui a cultivé au moins une parcelle pendant la périoxde 1993-1994. 25\% des personnes résidentes à Ekom sont des femmes cultivatrices (39 fenmes sur 41 femmes actives (> 15 ans)).

\section{LÉGEHDES DES PHOTOS}

\section{1: Tapis de Chromolaena odorata à Essienbot.}

L'ébour latjete (jachère préforestière) est dominé par le ('bromlatena odorala (Badjoué: Zclyt). A l'avant-plan, un champ dont les arachides viennent d'être récoltées. Le manioc reste. Certains arbres n'ont pas été ahattus; à gauche on voit le Pycnantbus angolemsis et à droite le Dislbemonanthus bentbamianus.

? : Défrichage du kwalkomo à Nbwampeuh (Ekom). Au premier plan, un gros parasolier (Musanga cecroptoides) à racines échasses qui doit encore être ahattu. Derrière, des bananeraies de marsavril 1993. La zone de Nbwampeult produit beaucoup de plantain.

3 : Plantation de rejets de plantain. L'homme défriche le kwalkomo tandis que son épouse plante les rejets de plantain. les arbres seront abattus et brûlés. Ce brûlage épargnera cependant le plantain déjà enfoui.

\section{4: Chanup du type "arachides» établi} sur ébour lalelelele.

Après la récolte des arachides, le plantain entre en compétition avec le manioc. À l'avant-plan, du macibo et de la canne à sucre

$\mathbf{S}$ : In endroit riclie en cendres. I es débris provenant du nettoyage du champ sont entassés et brilés. Cet endroit scera utilisé pour le semi de plantes exigentes, tels que tabac. amarante et tomate cerise. À côté du brûlis on trouve $\mathrm{Ic}_{\mathrm{c}}$ maniox, les atrachides et lea maïs qui constituent la matrice dominante du champ.

6. Sarclage des arachides. Ios femmes travaillent ensemble dans le champ de l'une d'entre elles. (ce champ est étahli sur de l'ébour latjetje.

\section{7: Champ de concombres.}

le Cucumeropsis mannii est très vigoureux. il grimpe sur la végétation abattue. On le récoltera en janvier.

8 : Conconibres presque mûrs. La plante a perdu son feuillage, certains fruits sont endommagés par les rongeurs.

9: Récolte des arachides. Les arachides sèchent sur des étagères ct des souches d'arbres à l'abri des rongeurs.

I0 : Barrière autsur du cacao. Pour limiter les dégâts occasionnés par les chimpanzés et les sitatungas, ce planteur construit une barrière haute de $1,80 \mathrm{~m}$. Il placera des pièges dans les ouvertures.

II : Athérure pris au piège. Ce rongeur (Atherurus africanus), de la famille des porcs-épics (IIystricickee), est un déprédateur du manioc et du cacao. Sa chair comestible est très appréciée.

\section{I2 : Maïs victime de rongeurs.} L'aulacode (Ibryonomys suinderianus) est probablement l'auteur de ces dégâts.

13: Panké autour d'un clamp. Cette barrière est faite avec des feuilles de palmier. Des pièges sont placés dans les ouvertures pour attraper des rongeurs et des reptiles (varans et serpents).

14 : Fabrication du piège upont». Efficace contre le singe moustac (Cercopithecus cephous), il protège les cacaoyères et les bananeraies.

15 : clôture de bois autour d'un champ d'arachides.

Le bois abattu dans la parcelle est érigé en clôture autour du champ pour former une barrière protectrice contre les déprédateurs. 